\title{
Fission observables from 4D Langevin calculations with macroscopic transport coefficients
}

\author{
Mark D. Usang ${ }^{1,4, \star}$, Fedir A. Ivanyuk ${ }^{1,2}$, Chikako Ishizuka ${ }^{1}$, Satoshi Chiba ${ }^{1,3}$, and Joachim A. \\ Maruhn ${ }^{5}$ \\ ${ }^{1}$ Tokyo Institute of Technology, Tokyo, Japan \\ ${ }^{2}$ Institute for Nuclear Research, Kiev, Ukraine \\ ${ }^{3}$ Astronomical Observatory of Japan, Tokyo, Japan \\ ${ }^{4}$ Malaysia Nuclear Agency, Bangi, Malaysia \\ ${ }^{5}$ Institut für Theoretische Physik, Frankfurt, Germany
}

\begin{abstract}
We have extended the Langevin equations to 4 dimensions (4D) by allowing the independent deformation for the left $\left(\delta_{1}\right)$ and right fragments $\left(\delta_{2}\right)$ of the fissioning nucleus. At the moment we are only able to use them in conjunction with the macroscopic transport coefficients. Nevertheless, we can see a considerable improvement in the preliminary results for the fission observables, especially those related to the total kinetic energy (TKE) of fission fragments. By plotting the TKE distributions we have revealed the super-long fission modes in ${ }^{236} \mathrm{U}$ and super-short fission modes in ${ }^{257} \mathrm{Fm}$. By plotting the distribution of $\delta$ against the fragment's TKE we have noted a correlation between the values of $\delta$ and Brosa's fission modes. We have found that the standard fission modes correspond to prolate tips of the light fragments while the complementary heavy fragments have oblate fission tips. On the other hand, if both fragments were prolate at the tips, we get super-long fission modes. If both fragments were oblate at the tips, we get super-short fission modes.
\end{abstract}

\section{Introduction}

The calculation of fission observables using Langevin equations has recently progressed from using 3D calculations to 4D. Previously [1], we constructed the 3D deformation potential within the two center shell model (TCSM) [2] and then allow the shape of the nucleus to evolve along the trajectories calculated by Langevin equations. The elongation of the nucleus was described by the normalized distance $z_{0} / R_{0}$ between the centers of the left and right potentials. Another collective coordinate, the mass asymmetry $\alpha=\left(A_{1}-A_{2}\right) /\left(A_{1}+A_{2}\right)$, was approximated by the asymmetry of the fragment's volume, $\alpha=\left(V_{1}-V_{2}\right) /\left(V_{1}+V_{2}\right)$. The fragments deformation is regulated mainly by the parameters $\delta_{i}$. In 3D calculations, we put in the $\delta_{1}=\delta_{2}$ restriction but for the current work on 4D calculations, we allow $\delta_{1}$ to change independently of $\delta_{2}$. Thus, in the current 4D calculations, the collective coordinates used in the Langevin calculations with macroscopic transport coefficients are $q_{i}=\left\{z_{0} / R_{0}, \delta_{1}, \delta_{2}, \alpha\right\}$.

^e-mail: usang.m.aa@titech.ac.jp; mark_dennis@nm.gov.my 
The Langevin equations [3] describe the changes of $q_{i}$ and their conjugate momenta $p_{i}$ with respect to time,

$$
\frac{d q_{i}}{d t}=\left(m^{-1}\right)_{i j} p_{j} ; \quad \frac{d p_{i}}{d t}=-\frac{\partial V\left(q, E_{i n t}\right)}{\partial q_{i}}-\frac{1}{2} \frac{\partial}{\partial q_{i}}\left(m^{-1}\right)_{j k} p_{j} p_{k}-\gamma_{i j}\left(m^{-1}\right)_{j k} p_{k}+g_{i j} R_{j}(t) .
$$

The inertia tensor $m_{i j}$ is calculated by the Werner-Wheeler method [4] and friction tensor $\gamma_{i j}$ is calculated by using the wall-and-window approach [5-9] with the reduction factor $k_{s}=0.27$. In the text, we refer to this inertia and friction tensor as macroscopic transport coefficients as it was inspired by hydrodynamical assumptions.

The thermal intrinsic energy, $E_{\text {int }}$ is estimated at each time step from the energy balance,

$$
E_{\text {int }}=E_{x}-\frac{1}{2}\left(m^{-1}\right)_{i j} p_{i} p_{j}-V(q, T=0)=a T^{2},
$$

where $E_{x}$ is the initial excitation energy of the system. In the case of neutron induced fission we assume $E_{x}=S_{n}+E_{n}$, where $S_{n}$ and $E_{n}$ are respectively the neutron separation energy and incident neutron kinetic energy. The temperature of the system is related to $E_{\text {int }}$ by the Fermi gas relation, $E_{\text {int }}=a T^{2}$, where $a$ is the level density parameter [10].

The strength of the random force $g_{i j}$ is related to the diffusion tensor, $D_{i j}=g_{i k} g_{j k}$ and for the diffusion tensor we used the modified Einstein relation, $D_{i j}=T^{*} \gamma_{i j}$. Here $T^{*}$ is the effective temperature $[11,12]$

$$
T^{*}=\frac{\hbar \varpi}{2} \operatorname{coth} \frac{\hbar \varpi}{2 T}, \quad \text { with } \hbar \varpi=2 \mathrm{MeV} .
$$

In the high temperature limit $T^{*}$ coincides with $T$. At low temperature the quantal aspects became dominant and the magnitude of diffusion coefficient becomes larger than those obtained from the classical Einstein relation. The parameter $\varpi$ is the local frequency of the collective motion and in principle it could be calculated at each deformation point. Unfortunately this would consume too much time. Hence, we simply use the constant value $\hbar \varpi=2 \mathrm{MeV}$. This value is close to the result from zero-th energy calculations [13]. In the limit of $T \rightarrow 0$, we have $T^{*} \rightarrow 1 \mathrm{MeV}$, but at high temperature limit, $T^{*} \rightarrow T$. 16],

The potential energy surface $V\left(q, E_{\text {int }}\right)$ is calculated by the macroscopic-microscopic method [14-

$$
V\left(q, E_{\text {int }}\right)=V_{\mathrm{LD}}^{\text {def }}(q)+\left[V_{\text {shell }}(q)+V_{\text {pair }}(q)\right] \Phi\left(E_{\text {int }}\right), \text { with } \quad \Phi\left(E_{\text {int }}\right)=e^{-E_{\text {int }} / E_{d}} .
$$

The macroscopic part of the potential energy $V_{\mathrm{LD}}^{\text {def }}(q)$ was calculated within the finite-range liquid drop model [17]. The energy and pairing shell corrections $V_{\text {shell }}(q)$ and $V_{\text {pair }}(q)$ at $T=0$ were calculated with the finite-depth deformed Woods-Saxon potential. For this the TCSM shapes were expressed in terms of deformed Cassini ovaloids and the code by Pashkevich $[18,19]$ was used to calculate the single-particle energies. The damping factors $\Phi\left(E_{\text {int }}\right)$ in present work was calculated by Ignatyuk prescription [20] with the shell damping energy $E_{d}=20 \mathrm{MeV}$.

We start the Langevin calculations as far inside the saddle as possible in order to account for the stochastic fluctuation in the equilibrated medium of nuclear collective motion inside the saddle. As the nucleus evolves, we check the radius of the neck, $R_{\text {neck}}$. Once the condition $R_{\text {neck }}=0$ is fulfilled, we consider such event as a fission event. The value of $\alpha$ at this configuration is extracted and the pre-neutron emission fragments mass are obtained from the formula, $A_{i}^{\mathrm{pre}}=0.5 A_{C N}\left(1+(-1)^{i-1} \alpha\right)$ for $i=\{1,2\}$ in reference to the left and right fission fragment, respectively. $A_{C N}$ is the compound nucleus mass, which is here either 236 or 257 . Hence, we are able to calculate the post-neutron emission mass by,

$$
A_{i}^{\mathrm{post}}=A_{i}^{\mathrm{pre}}-\bar{v}\left(A^{\mathrm{pre}}\right) .
$$


Theory-4

In present work we simply use the experimental results for $v\left(A^{\text {pre }}\right)$ from the reactions with the incident neutron energy $E_{n}=0.5 \mathrm{MeV}$ [21].

\section{Numerical Results}

The preliminary results of our calculations for ${ }^{236} \mathrm{U}$ and ${ }^{257} \mathrm{Fm}$ are shown in Fig. 1 and Fig. 2, respectively. In each figure, the fission fragment mass yield and total kinetic energy (TKE) distribution are shown. In each of these figures, we plot the pre-neutron emission fission fragment yield from our calculation. In the case of ${ }^{236} \mathrm{U}$, we plot also the estimated post-neutron emission fission fragment yield from the calculated pre-neutron yield.
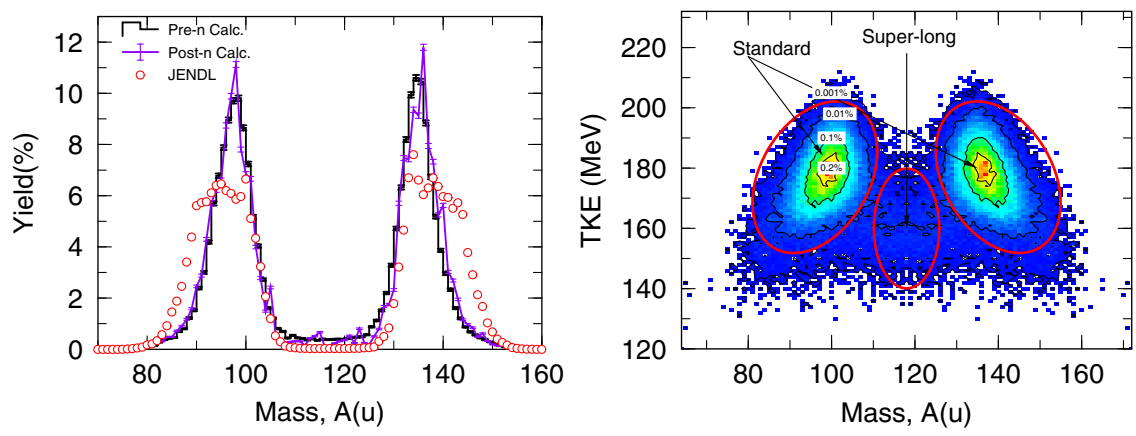

Figure 1. The calculated fission fragment yield of ${ }^{236} \mathrm{U}$ at $E_{x}=7 \mathrm{MeV}$ on the left, and TKE on the right. On the left figure, pre-neutron emission fission fragment (Pre-n Calc.) and post-neutron emission fission fragment (Post-n Calc.) are compared with the JENDL thermal fission product yield. The post-neutron emission fission fragment mass is calculated according to Eq. (5). On the right figure, we mark Brosa's standard fission modes (Standard) and super-long fission modes (Super-long).
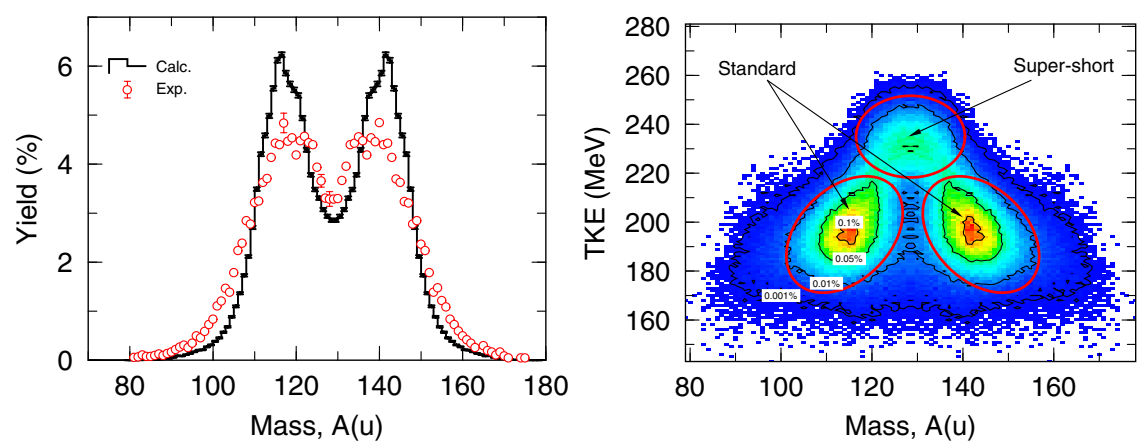

Figure 2. The calculated fission fragment yield of ${ }^{257} \mathrm{Fm}$ at $E_{x}=7 \mathrm{MeV}$ on the left, and TKE on the right. The pre-neutron emission fission fragment yield (Calc.) is compared with the experimental [22] spontaneous fission data (Exp.). We mark the presence of standard and super-short fission modes on the TKE figure.

Both calculations for ${ }^{236} \mathrm{U}$ and ${ }^{257} \mathrm{Fm}$ are done at $E_{x}=7 \mathrm{MeV}$ and we see that the average mass of the light and heavy fragments align with the estimates from either evaluated or experimental yields. 
More precisely, in the case of ${ }^{236} \mathrm{U}$, the pre-neutron emission light fission fragment mass average is 99.17 and the heavy fission fragment mass average is 136.83 . After neutron emission, the light and heavy fragment mass average are 97.14 and 135.59 each. This should be compared with the evaluated data from JENDL [23] - the average mass of 94.75 and 138.68 for the light and heavy fission fragment, respectively. In the case of ${ }^{257} \mathrm{Fm}$, the average mass for light and heavy fragments are 116.40 and 140.60. The experimental mass averages [22], 115.41 and 141.40 each for light and heavy fission fragments, are very close to our calculations. This clearly implies that the potential surface is correct or at least the position of the potential minimum or saddle is correct.

However, the peaks are thinner and too tall. It seems the macroscopic transport coefficients used in present work are not so good.

\subsection{Distributions of TKE}

Regarding the TKE, on the other hand, the general shape and distribution are quite good. The TKE distribution on both ${ }^{236} \mathrm{U}$ and ${ }^{257} \mathrm{Fm}$ on Figs. 1 and 2 clearly reveals the standard fission modes. In ${ }^{236} \mathrm{U}$, the standard fission modes are prevalent regardless of the fission fragments TKE. According to Brosa et al. [24] these fission modes are formed by the prescission shapes of the nucleus. The standard fission modes are described to be slightly asymmetric and of "standard" length. Indeed, in the case of ${ }^{236} \mathrm{U}$, the average length measured in terms of center of mass distance $\left\langle R_{12}\right\rangle$ is around 2.5 to 2.6 times larger than the radius of spherical nucleus $R_{0}$ for the fragments with $A \leqslant 110$ and $A \geqslant 130$.
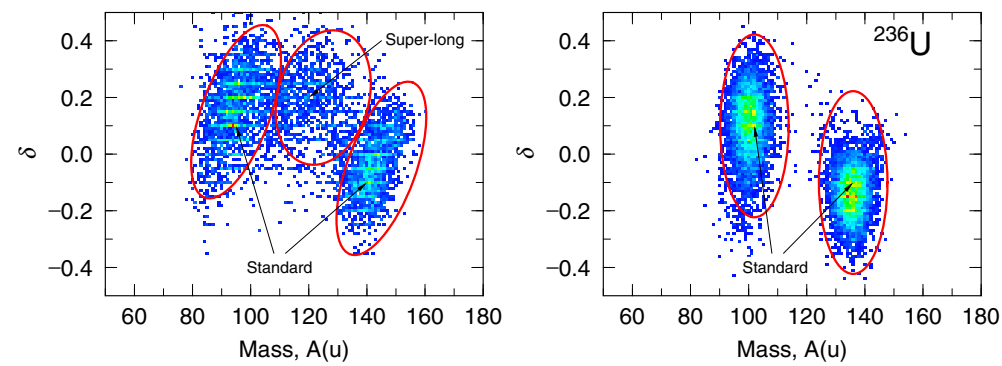

Figure 3. The distribution of deformation parameter $\delta_{i}$ calculated at $E_{x}=7 \mathrm{MeV}$ for ${ }^{236} \mathrm{U}$ at the scission configuration with low TKE $(160 \mathrm{MeV} \leq \mathrm{TKE} \leq 165 \mathrm{MeV}$, left $)$ and high TKE $(185 \mathrm{MeV}<\mathrm{TKE} \leqslant 190 \mathrm{MeV}$, right). We indicate the emerging Brosa's fission modes.

In Fig. 3 we show the distribution of parameter $\delta$ at the scission point. One can see the clusters of fission fragments with an asymmetric mass around $\delta \approx 0.2$ for the lighter fragments and $\delta \approx-0.1$ for the heavy fragments. The value of $\delta$ describes the shapes of tips of the fissioning nuclei. Positive $\delta$ corresponds to a prolate tip, negative $\delta$ corresponds to oblate tip shape and $\delta=0$ means that the tips are spherical. It is easy to infer from here, that if the tips of both fragments are prolate, we will get a longer prescission nucleus as it was stretched apart. If they are both oblate, the prescission fragments would be closer to each other in order to conserve the volume. The long prescission shapes are described by Brosa as the super-long fission modes and the short prescission shapes are called super-short fission modes.

We can observe the super-long fission modes in ${ }^{236} \mathrm{U}$ from Figs. 1 and 3 at symmetric mass around $110 \leqslant A \leqslant 130$. The fragments at these symmetric mass mostly appear at TKE lower than $180 \mathrm{MeV}$. In Fig. 3 , it is clear that it clusters around $\delta \approx 0.2$ also, but because it was of symmetric mass, both complementary fragments would be similarly prolate at the tips. The final confirmation of this fission 
Theory-4

modes came from the length of the prescission shapes, yielding $\left\langle R_{12}\right\rangle$ of approximately $2.8 R_{0}$ to $2.9 R_{0}$. This is far longer than the prescission shape we see for the standard fission modes of ${ }^{236} \mathrm{U}$.

In ${ }^{257} \mathrm{Fm}$ the standard fission modes have prescission shapes that are slightly longer than that for ${ }^{236} \mathrm{U},\left\langle R_{12}\right\rangle \approx 2.7 R_{0}$. The TKE of the standard fission modes however only gets as large as $220 \mathrm{MeV}$, as we can see from Fig. 2. In fact, as we can see from Fig. 4, the standard fission modes of ${ }^{257} \mathrm{Fm}$ are present only at low TKE and completely absent from contributing to high TKE fission fragments. Similar to ${ }^{236} \mathrm{U}$ standard fission modes, in ${ }^{257} \mathrm{Fm}$ the light fragments are prolate and the heavy fragments are oblate at the tips. At high TKE of around $235 \mathrm{MeV}$, only fission fragments from the super-short fission modes can be seen. At such high TKE, we see that the fission fragments clustered around symmetric fragment mass with very oblate fissioning shapes at $\delta \approx-0.2$. As such, the length of the prescission shape, $\left\langle R_{12}\right\rangle$ is less than $2.45 R_{0}$ at the symmetric mass of $\mathrm{A}=128$.
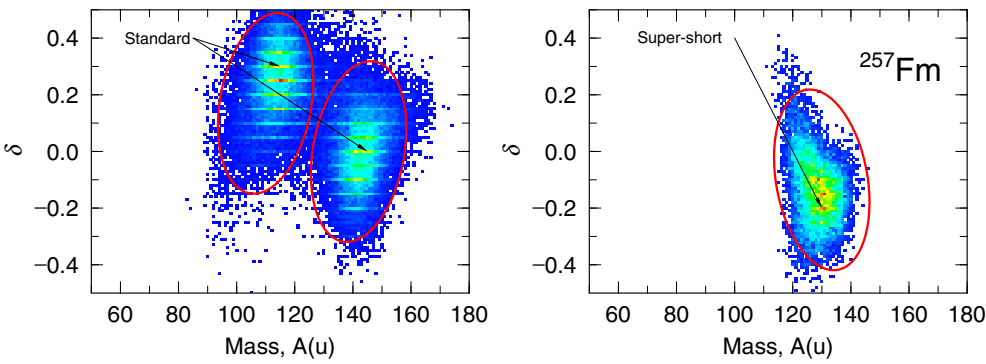

Figure 4. The distribution of deformation parameter $\delta_{i}$ calculated at $E_{x}=7 \mathrm{MeV}$ for ${ }^{257} \mathrm{Fm}$ at scission that has low TKE $(190 \mathrm{MeV} \leq \mathrm{TKE} \leq 195 \mathrm{MeV}$, left $)$ and high TKE $(235 \mathrm{MeV}<\mathrm{TKE} \leqslant 240 \mathrm{MeV}$, right). We indicate also the emerging Brosa's fission modes.

\section{Conclusion}

The systematics by Viola [25] predicts the TKE for ${ }^{236} \mathrm{U}$ and ${ }^{257} \mathrm{Fm}$ at $170.15 \mathrm{MeV}$ and $194.31 \mathrm{MeV}$, respectively. Both these estimates deviate from the corresponding experimental TKE. In the case of ${ }^{236} \mathrm{U}$, the systematics slightly overestimates the TKE for thermal fission which gave the value around $169.71 \mathrm{MeV}$, according to JENDL. On the other hand, Viola systematics underestimates the TKE for ${ }^{257} \mathrm{Fm}$ experimental TKE [26] of $197.6 \mathrm{MeV}$. This deviation can be caused by the presence of either super-long or super-short fission modes, if the Brosa systematic only tracks TKE from standard fission modes. Our calculations, however, are unable to further clarify this problem at the moment because the average TKE in our calculation is too high at $176.59 \mathrm{MeV}$ and $202.55 \mathrm{MeV}$, respectively, for ${ }^{236} \mathrm{U}$ and ${ }^{257} \mathrm{Fm}$ due to the slightly poor mass yield. If we were able to match either the evaluated or experimental average TKE by fixing the yield, we would probably be able to examine the validity of this question by removing the contribution from symmetric yield.

In the present study, the shape of the tips seems to be inherent in allowing for further lengthening or shortening of prescission shape. If the tips are prolate, the shape at the scission point is more elongated and the Coulomb interaction energy between fragments - smaller, and oblate shapes lead to a larger Coulomb interaction energy.

It would also be interesting to expand the current work to consider the fission observables from ${ }^{258} \mathrm{Fm}$ and other nuclides of interest. 
Recently we have submitted a more detailed paper [27] on the fission observables from ${ }^{236} \mathrm{U}$ using the 4D Langevin approach with macroscopic transport coefficients and some other improvements.

The present work included the results for Comprehensive study of delayed-neutron yields for accurate evaluation of kinetics of high-burn up reactors entrusted to Tokyo Institute of Technology by the Ministry of Education, Culture, Sports, Science and Technology of Japan (MEXT).

\section{References}

[1] M.D. Usang, F.A. Ivanyuk, C. Ishizuka, S. Chiba, Phys. Rev. C 94, 044602 (2016)

[2] J. Maruhn, W. Greiner, Z. Phys. 251, 431 (1972)

[3] Y. Abe, S. Ayik, P.G. Reinhard, E. Suraud, Phys. Rep. 275, 49 (1996)

[4] K.T.R. Davies, A.J. Sierk, J.R. Nix, Phys. Rev. C 13, 2385 (1976)

[5] J. Blocki, Y. Boneh, J. Nix, J. Randrup, M. Robel, A. Sierk, W. Swiatecki, Ann. Phys. 113, 330 (1978)

[6] G. Adeev, A. Karpov, P. Nadtochii, D. Vanin, Phys. Part. Nucl. 36, 378 (2005)

[7] H.J. Krappe, K. Pomorski, Theory of Nuclear Fission: A Textbook, Vol. 838 (Springer Science \& Business Media, 2012)

[8] W. Swiatecki, Nucl. Phys. A 428, 199 (1984)

[9] J.R. Nix, A.J. Sierk, Nucl. Phys. A 428, 161 (1984)

[10] J. Tōke, W. Światecki, Nucl. Phys. A 372, 141 (1981)

[11] H. Hofmann, D. Kiderlen, Int. J. Mod. Phys. E 07, 243 (1998)

[12] H. Hofmann, C. Grégoire, R. Lucas, C. Ngô, Z. Physik A 293, 229 (1979)

[13] D.L. Hill, J.A. Wheeler, Phys. Rev. 89, 1102 (1953)

[14] V. Strutinsky, Nucl. Phys. A 95, 420 (1967)

[15] V. Strutinsky, Nucl. Phys. A 122, 1 (1968)

[16] M. Brack, J. Damgaard, A.S. Jensen, H.C. Pauli, V.M. Strutinsky, C.Y. Wong, Rev. Mod. Phys. 44, 320 (1972)

[17] H.J. Krappe, J.R. Nix, A.J. Sierk, Phys. Rev. C 20, 992 (1979)

[18] V. Pashkevich, Nucl. Phys. A 169, 275 (1971)

[19] V. Pashkevich, A. Rusanov, Nucl. Phys. A 810, 77 (2008)

[20] A. Ignatyuk, K. Istekov, G. Smirenkin, Sov. J. Nucl. Phys. 29, 450 (1979)

[21] R. Müller, A.A. Naqvi, F. Käppeler, F. Dickmann, Phys. Rev. C 29, 885 (1984)

[22] J.P. Balagna, G.P. Ford, D.C. Hoffman, J.D. Knight, Phys. Rev. Lett. 26, 145 (1971)

[23] K. Shibata, O. Iwamoto, T. Nakagawa, N. Iwamoto, A. Ichihara, S. Kunieda, S. Chiba, K. Furutaka, N. Otuka, T. Ohasawa et al., J. Nucl. Sci. Technol. 48, 1 (2011)

[24] U. Brosa, S. Grossmann, A. Müller, Phys. Rep. 197, 167 (1990)

[25] V.E. Viola, K. Kwiatkowski, M. Walker, Phys. Rev. C 31, 1550 (1985)

[26] D.C. Hoffman, M.R. Lane, Radiochim. Acta 70, 135 (1995)

[27] C. Ishizuka, M.D. Usang, F.A. Ivanyuk, J.A. Maruhn, K. Nishio, S. Chiba (submitted to Phys. Rev. C) 